\title{
Informe del grupo de expertos ad hoc sobre la transición de la asistencia institucional a la de base comunitaria1,2
}

\author{
Dirección General de Empleo, \\ Asuntos Sociales e Igualdad de Oportunidades \\ Comisión Europea
}

Zantzu argiak aurkitu izan dira asistentzia instituzionalaren ordezko aukera komunitarioen alde egiteko (desinstituzionalizazioa). Izan ere, aukera komunitario horiek emaitza hobeak eskaini omen ditzakete erabiltzaile, senide eta langileentzat; eta, gainera, antzekoak omen dira arreta modu horretarako kostuak eta ohiko asistentzia instituzionalarenak. Artikulu honetan, saiatu dira balizko erronka zailenak identifikatzen baliabide instituzionalak uzterako prozesu horretan, eta proposatzen dituzte oinarrizko printzipio gidariak trantsizio bide hori egiteko, betiere, aipatutako erronken ebaluatzean eta eremu honetan bultzatutako jarduera egokienak oinarri hartuz. Europako Batzordeak argitara emandako dokumentu honetako gomendioak berariaz zuzendu dituzte Europar Batasuneko Estatu-kide eta Europako Batzordearentzat.

\section{HITZ-GAKOAK:}

menpekotasuna, ezgaitasuna, desinstituzionalizazioa, gomendioak, Europar Batasuna.
Existen indicios sólidos que apoyan la transición de la asistencia institucional a las alternativas de base comunitaria (desinstitucionalización), ya que pueden reportar mejores resultados a los usuarios, sus familias y el personal, siendo sus costes equiparables a los de la asistencia institucional. Este artículo identifica algunos desafíos clave para los procesos de desinstitucionalización y propone un conjunto de principios básicos comunes para guiarlos, que se basan tanto en la evaluación de los citados desafíos, así como en las mejores prácticas desarrolladas en este ámbito. Las recomendaciones se dirigen tanto a los Estados miembros de la Unión Europea, como a la propia Comisión Europea, editora del documento.

\section{PALABRAs CLAVE:}

dependencia, discapacidad, desinstitucionalización, recomendaciones, Unión Europea.

${ }^{1}$ El grupo de expertos ad hoc estaba formado por: Jan Pfeiffer (presidente) y Georgette Mulheir, del Children's High Level Group; AnneSophie Parent, de la European Older People's Platform (AGE); Céline Simonin, de la Confederation of Family Organisations in the EU (COFACE); Luk Zelderloo, de la European Association of Service Providers for Persons with Disabilities (EASPD); Ines Bulic, de la European Coalition for Community Living (ECCL); Carlotta Besozzi y Ask Andersen, del European Disability Forum (EDF); Geert Freyhoff, de Inclusion Europe; y Josée van Remoortel, de Mental Health Europe. El grupo contó con la asistencia de una secretaría formada por Jan Jařab y Silvio Grieco, de la Comisión Europea.

2 Se reproduce aquí el siguiente documento: Dirección General de Empleo, Asuntos Sociales e Igualdad de Oportunidades, Informe del grupo de expertos ad hoc sobre la transición de la asistencia institucional a la de base comunitaria, Bruselas, Comisión Europea, 20o9. Se trata de un informe traducido por la propia Comisión, a partir de un original en inglés. Para ajustarlo al formato de artículo, se han suprimido los agradecimientos y el índice, se ha adaptado y recortado el resumen ejecutivo, se han añadido los descriptores, se ha elaborado una bibliografía con los recursos documentales citados y se ha procedido a algunos ajustes formales. Por lo demás, se publica el texto en su integridad. (C) Comunidades Europeas, 2009. Reproducción autorizada, con indicación de la fuente bibliográfica. 


\section{Prólogo3}

Son muchos los ciudadanos de toda la Unión Europea que pasan largos años de su vida en instituciones de asistencia. Algunos tienen alguna discapacidad física o intelectual, otros sufren problemas de salud mental y otros son demasiado mayores y delicados. También hay muchos niños internados en instituciones, niños con y sin discapacidad.

Durante varias décadas, la existencia de dicha asistencia institucional se ha considerado una prueba de que la sociedad se preocupa, de que no deja a las personas vulnerables sin asistencia y de que proporciona alimentos, cobijo, ropa y tratamientos a las personas necesitadas. Pero ¿acaso es éste el mejor modelo que pueden ofrecer las sociedades europeas avanzadas a estas personas en el siglo XXI? Estoy convencido de que en una era en la que los aspectos inmateriales tales como la dignidad humana, la autonomía y la inclusión en la comunidad cada vez están adquiriendo mayor importancia, las sociedades europeas deberían buscar modelos de asistencia más humanos, centrados en las personas e individualizados. Se debe asociar y hacer partícipes de la toma de decisiones a los propios usuarios y, en la medida de lo posible, también sus familias. Todo el mundo debería ser capaz de alcanzar su máximo potencial.

La Comisión Europea ha financiado estudios anteriores que contenían comparaciones entre la asistencia institucional y la de base comunitaria en cuanto a calidad y costes. La labor del grupo ad hoc de expertos sobre la transición de la asistencia institucional a la de base comunitaria va un paso más allá. No se limita a ofrecer un razonamiento, bien argumentado, del cambio, sino que ofrece además asesoramiento real sobre el modo de alcanzar dicha transición (y sobre qué debería evitarse), derivado de la experiencia adquirida en aquellos Estados miembros en los que ya se han acometido este tipo de reformas. El análisis de los desafíos fundamentales, seguido de un conjunto de Principios Básicos Comunes y una serie de recomendaciones concretas dirigidas a los Estados miembros y a la Comisión Europea podrían verse como la 'combinación perfecta' para alcanzar unas aspiraciones idealistas con unas directrices concretas y pragmáticas.

Las cuestiones que se abordan en el presente informe son inherentes a nuestro compromiso europeo conjunto con los valores de los derechos fundamentales y la inclusión social. Por todo ello, acojo con satisfacción el resultado del trabajo de este grupo de expertos, un informe breve y conciso, y espero que se convierta en fuente de inspiración para los

${ }^{3}$ A cargo de Vladimír Špidla, comisario de Empleo, Asuntos Sociales e Igualdad de Oportunidades. responsables políticos de los Estados miembros y del ámbito europeo.

\section{Marco conceptual}

\subsection{El derecho a vivir de forma independiente y a ser incluido en la comunidad}

La Unión Europea y sus Estados miembros se fundamentan en los valores comunes de respeto de la dignidad humana, libertad, democracia, igualdad, Estado de Derecho y respeto de los derechos humanos. Todas las personas tienen el derecho a vivir de forma independiente y a ser incluidas en la comunidad, el derecho a elegir su lugar de residencia y dónde y con quién vivir, y el derecho a vivir con dignidad. Todos los niños tienen derecho a la vida familiar, ya sea en su propia familia o en una familia de acogida (si carecen de familia o si la permanencia en su familia constituye algún riesgo para ellos). Estos derechos se recogen en los correspondientes tratados internacionales (Naciones Unidas) y europeos sobre derechos humanos, entre ellos, la Convención sobre los Derechos de las Personas con Discapacidad4, la Convención sobre los Derechos del Niño, la Carta Social Europea revisada 5 y la Carta de los Derechos Fundamentales de la Unión Europea.

En la práctica, el derecho a vivir en la comunidad pasa por la disponibilidad de una serie de servicios de base comunitaria que brinden a las personas el apoyo que necesiten y les permitan participar en la vida cotidiana. Por contra, cabría considerar que el internamiento de larga duración de las personas en instituciones residenciales está reñido con su derecho a vivir en la comunidad.

\subsection{Los usuarios de los servicios}

Tanto los marcos legislativos como las investigaciones académicas suelen aplicar enfoques distintos a las cuatro categorías de usuarios de estos servicios: las personas con discapacidad, los ancianos, los niños y las personas con problemas de salud mental. El objetivo del presente informe consiste en proporcionar un enfoque transversal, procurando subrayar las características y los enfoques comunes.

Sin embargo, es sabido que las soluciones propuestas han de adaptarse a las necesidades inherentes a cada una de las cuatro categorías. Por ejemplo, ya se sabe que los niños, tanto los que tienen discapacidad como los que no, son particularmente vulnerables a los efectos perjudiciales del internamiento en instituciones debido a que éstas no proporcionan un

\footnotetext{
4 Véanse, en particular, los artículos 12, 14, 15, 16, 17, 19, 23 , 25,26 y 28

5 Véanse, en particular, los artículos 15, 17 y 23.
} 
entorno adecuado para su desarrollo moral, psicológico ni emocional.

\subsection{Definición de las instituciones}

No resulta fácil proporcionar una definición genérica de una 'institución' ni de la 'asistencia institucional' debido, principalmente, a los distintos marcos culturales y jurídicos de los Estados miembros. En algunos de ellos, por ejemplo, como consecuencia de sus marcos legales, se consideran 'instituciones' incluso a los proveedores de servicios no residenciales de base comunitaria. No obstante, para los fines del presente informe, por 'asistencia institucional' entenderemos la asistencia brindada en instituciones residenciales segregadoras.

Algunas definiciones de instituciones se basan en el número de plazas de las instalaciones, es decir, definen una institución como una instalación residencial con treinta plazas o más. Esta definición puede resultar de utilidad a la hora de recopilar datos, describir tendencias y hacer un seguimiento del progreso, pero una institución no se define exclusivamente por su tamaño, el cual no constituye sino un mero indicador de una serie de características más esenciales que hacen que un servicio sea 'institucional'. Es cierto que da la impresión de que cuanto mayor es el emplazamiento menos posibilidades existen de garantizar unos servicios individualizados y personalizados y una participación o una inclusión en la comunidad. Por otra parte, la situación contraria no es necesaria ni inversamente proporcional (es decir, pueden existir instalaciones de menor tamaño que brinden una asistencia de carácter profundamente institucional).

\subsection{La cultura institucional}

En lugar de utilizar un punto de corte específico para determinar el tamaño de las instituciones, en el presente informe se abordarán los conceptos de 'institución' o ‘asistencia institucional' desde el punto de vista de los derechos humanos y la dignidad de los usuarios, su calidad de vida y su salud, su autonomía y su inclusión social. Cuando hablemos de 'instituciones' nos referiremos a los servicios de asistencia residencial que practiquen una 'cultura institucional'; las características exteriores de las instituciones -los edificios en sí- se consideran meramente los factores más visibles (pero no necesariamente los más importantes) entre los factores definitorios.

Las características típicas de la 'cultura institucional' fueron descritas y analizadas por una serie de investigadores precursores hace aproximadamente cuatro décadas. Durante mucho tiempo, se ha sostenido la idea de que la asistencia en instituciones segrega a los usuarios y suele estar caracterizada por una despersonalización (eliminación de las posesiones personales, las señales y los símbolos de individualidad e humanidad), una rigidez en las rutinas (horarios fijos para levantarse, comer y realizar actividades independientemente de las preferencias 0 las necesidades individuales), un trato en bloque (atendiendo a las personas como miembros de un grupo sin privacidad ni individualidad) y una distancia social (poniendo de manifiesto las diferencias en el estatus del personal y los residentes) [King, Raynes y Tizard, 1971]. Los residentes en este tipo de instituciones desarrollan una conducta pasiva ('institucionalizada') al adaptarse a estas rutinas, al aburrimiento y a la falta de actividades significativas. Asimismo, los profesionales que prestan este tipo de asistencia -como muy bien describió Goffman (1968) en el caso de una institución de salud mental- tienden a estar cada vez más 'institucionalizados' con el paso del tiempo.

En última instancia, el ejercicio de la asistencia es reflejo de las actitudes y las creencias exigidas por la institución al personal para hacer frente a las tareas determinadas por esta misma. Tradicionalmente, la ética que rodea a la asistencia ha sido paternalista en lugar de interactiva. En varios tipos de instituciones (para personas con discapacidad y para quienes padecen problemas de salud mental y, en cierto modo, también en las instituciones para ancianos y en las infantiles), el predominio de profesionales médicos y personal de atención sanitaria sigue fomentando una distancia social entre el personal y los usuarios. El modelo médico de la asistencia entraña el riesgo de reducir a los individuos a sus diagnósticos.

Para los fines del presente informe se entenderá por 'asistencia institucional' cualquier tipo de asistencia residencial en que:

- Los usuarios estén aislados del resto de la comunidad u obligados a vivir juntos.

- Estos usuarios no posean un control suficiente sobre su vida ni sobre las decisiones que les afecten.

- Los requisitos de la organización tiendan a primar sobre las necesidades individuales de los usuarios $^{6}$.

\footnotetext{
${ }^{6}$ Los autores se basan en parte en la definición que ofrece de un grupo de usuarios específico -las personas con deficiencia mental- una organización activista canadiense, People First: una institución es cualquier lugar en el que se aísle, segregue o congregue a personas que hayan sido etiquetadas como personas con deficiencia mental. Una institución es todo lugar en el que las personas no posean control sobre sus vidas ni sobre sus decisiones diarias, o no se les permita ejercerlo. Una institución no se define simplemente en función de su tamaño.
} 


\section{La situación actual en la UE}

El Informe conjunto sobre protección e inclusión social (Comisión Europea, 2009) más reciente de la UE, que fue presentado en 2009, pone de manifiesto la necesidad de reiterar el compromiso de garantizar un acceso universal a la asistencia de larga duración de gran calidad y al alcance de todos. Aunque la mayor parte de las personas que necesitan apoyo prefieren recibir estos servicios en su hogar o en un entorno de base comunitaria en lugar de en una institución, en muchos países la asistencia institucional sigue representando más de la mitad del gasto público en este ámbito. Si bien este patrón es más acusado en los países de Europa central y oriental, no se trata, en absoluto, de una característica exclusiva de los mismos. En algunos Estados miembros de la UE, unas normas administrativas y jurídicas excesivamente rígidas (por ejemplo, relativas a la financiación, a la seguridad o la higiene) dificultan la prestación de servicios en entornos distintos a las grandes instituciones.

El informe derivado de un estudio realizado en los Estados miembros de la UE y Turquía, De-institutionalisation and Community Living: Outcomes and Costs (en adelante DECLOC) [Mansell et al., 2007], señaló que casi 1,2 millones de niños y adultos con discapacidad vivían en instituciones residenciales de estancia prolongada. Más de una cuarta parte de las plazas de las instituciones las ocupan personas con deficiencia mental, siendo el siguiente grupo más representado el de las personas con problemas de salud mental. Es previsible que el número total de residentes sea incluso mayor, dado que la mayor parte de los Estados miembros solamente conservan datos parciales sobre el número de personas internadas en las instituciones. El DECLOC señaló que, en 16 de los 25 países de los que había información disponible, se utilizan fondos estatales (locales o regionales), al menos parcialmente, para apoyar a las instituciones con más de 100 plazas. En 21 países se utilizan fondos estatales para apoyar a las instituciones con más de 30 plazas.

En el ámbito de la salud mental, se ha registrado un descenso acusado de la asistencia institucional de larga duración durante las últimas décadas. Esta tendencia forma parte de una reducción general del número de camas en los hospitales psiquiátricos, tal y como pone de manifiesto el informe MHEEN II (Medeiros et al., 2008). Sin embargo, el alcance de estos cambios varía considerablemente, siendo mucho más notable en Europa occidental que en Europa central y oriental.

Por lo que respecta a los niños, se calcula que en la UE7 150.000 niños viven en entornos de asistencia

7 Según una reciente recopilación de estudios nacionales sobre la situación en la UE realizada por Eurochild (2009). residencial, entre ellos, 'escuelas especiales', casas cuna, centros para personas con deficiencia mental o con discapacidad física, centros para niños con problemas de conducta, instituciones para delincuentes juveniles y centros de reinserción. En la mayor parte de los países de Europa occidental, la mayoría de los niños que reciben asistencia pública se colocan en entornos de base familiar, frente a la colocación en familias de acogida o con parientes. En Europa central y oriental la situación difiere, predominando todavía los entornos de asistencia residencial.

No obstante, estos datos siguen siendo parciales y fragmentados, lo que se traduce en que actualmente no es posible ofrecer una visión global.

\section{Argumentos a favor de la transición de la asistencia institucional a la de base comunitaria}

\subsection{Aspectos materiales e inmateriales de la asis- tencia en instituciones}

Las condiciones materiales de la vida en las instituciones tienden a ser peores que las de la mayoría de los miembros de la sociedad en general. Además, las cuatro características 'clásicas' - despersonalización, rigidez en las rutinas, trato en bloque y distancia social- suelen ser especialmente acusadas cuando las condiciones son deficientes: si la dirección tiene dificultades para garantizar alimentos, calefacción y otras necesidades básicas, la terapia o las actividades significativas apenas tienen cabida. Recientemente se han documentado casos extremos de privación material combinados con prácticas negligentes o abusivas, especialmente en algunos ‘nuevos' Estados miembros, aunque también en los 'antiguos'.

No obstante, las características problemáticas de la asistencia institucional no están exclusivamente vinculadas a unas condiciones materiales deficientes, y no es seguro que puedan resolverse simplemente con la mejora de las mismas. Obviamente, la calidad de la asistencia se puede mejorar a través de mejores ratios entre personal y usuarios y un mayor incremento de la atención dedicada a actividades significativas. Sin embargo, sigue habiendo problemas vinculados a la despersonalización, el trato en bloque, la rigidez de las rutinas y la distancia social también en aquellos establecimientos en los que las condiciones materiales son razonablemente óptimas.

Además, crece la tendencia a considerar algunas de las características inherentes a las instituciones como estigmatizadoras de los usuarios que se ven física y socialmente aislados (segregados) del resto de la sociedad. Tanto si es por motivos políticos 0 por el deseo de acceder a fuentes de ayuda alterna- 
tivas, la mayoría de los residentes tienen dificultades a la hora de abandonarlas para vivir en otro lugar, lo cual, una vez más, parece ser un problema inherente a la asistencia institucional que no puede solventarse con un aumento del gasto destinado a las instituciones.

La calidad de las instituciones contemporáneas varía enormemente entre los Estados miembros y dentro de determinados países en términos de tamaño, condiciones materiales y ejercicio de la asistencia. Por consiguiente, estas instituciones manifiestan las citadas características en distinta medida. Sin embargo, se puede argumentar que algunos problemas son, en principio, inherentes al sistema institucional en sí. Las investigaciones disponibles están muy centradas en países de habla inglesa, pero un estudio reciente (Freyhoff et al., 2004) de las instituciones en Francia, Hungría, Polonia y Rumanía puso de manifiesto que, si bien existían diferencias entre las instituciones de dichos Estados miembros, los resultados eran igualmente deficientes en lo referente a los residentes y las instituciones que los estudiados en el Reino Unido, los Estados Unidos y Suecia en la década de los años setenta.

Por tanto, el modelo institucional de asistencia cada vez se considera más inadecuado. Existe una creencia incipiente -aunque tal vez esté falta de un consenso claro- de que independientemente de la cantidad de dinero que se gaste en las instituciones, las características de la asistencia institucional seguro que dificultan la prestación de una calidad de vida adecuada a los usuarios, el aseguramiento del disfrute de los derechos humanos y la consecución del objetivo de inclusión social.

\subsection{La asistencia institucional frente a la vida en la comunidad}

Si bien es cierto que en algunos Estados miembros o en sus regiones las grandes instituciones preexistentes fueron sustituidas, en su mayoría, por instituciones de menor tamaño, en otros se ha producido una auténtica desinstitucionalización, con la sustitución gradual de las grandes instituciones por servicios de apoyo en la comunidad (asistencia comunitaria, asistencia domiciliaria). Entre los precursores de la desinstitucionalización cabe mencionar a Suecia y al Reino Unido, así como a países no miembros a la UE tales como Noruega o los Estados Unidos.

Los indicios recabados de la investigación y la evaluación de alternativas a la asistencia institucional apoyan la transición a unos servicios de base comunitaria (informe DECLOC). Un gran número de estudios de investigación arrojan, predominantemente, mejores resultados con respecto a las personas que reciben los servicios, sus familias y el personal que las apoya.
Una revisión de 73 estudios de desinstitucionalización y vida en la comunidad realizados a mediados de la década de los años noventa (Emerson y Hatton, 1994) puso de relieve la variedad de los servicios en un espectro de medidas distintas. Los datos que contenían demostraban que los rangos de puntuación de las distintas medidas se solapaban considerablemente entre las grandes instituciones, las instituciones pequeñas y los servicios de base comunitaria. En término medio, los servicios de base comunitaria se perfilaban como la mejor opción. En una evaluación del efecto de la transición de los servicios institucionales a los comunitarios en el Reino Unido e Irlanda, la mayoría de los estudios mostraban efectos positivos en cinco o seis esferas (competencia y crecimiento personal, conducta desafiante observada, participación en la comunidad, participación en actividades significativas y contacto por parte del personal); sólo hubo una esfera (conducta desafiante notificada) en la que casi ningún estudio demostró ninguna variación. Los estudios realizados en los Estados Unidos y en Australia arrojan resultados similares (aunque ligeramente menos definidos).

\section{El modelo institucional}

\section{cada vez se considera más inadecuado. Además, la mayoría de los usuarios, antes que en instituciones prefieren vivir en la comunidad}

En términos generales, los estudios disponibles confirman que si se prestan unos servicios comunitarios de calidad, la mayor parte de los usuarios antes internados en instituciones muestran una preferencia clara por la vida en la comunidad y presentan índices más elevados de satisfacción personal e inclusión social, existiendo menos problemas vinculados a la inseguridad o a la soledad de lo previsto. Included in Society (Dentro de la sociedad), un estudio con recomendaciones financiado por la Comisión Europea en el que se describían los problemas generales de la asistencia institucional así como casos de éxito relacionados con la desinstitucionalización, concluía que, en término medio, los servicios de base comunitaria ofrecen a las personas con discapacidad mejores resultados que las instituciones en lo referente a la calidad de vida, a la vez que admitía que la sustitución de las instituciones por alternativas de base comunitaria no garantiza de por sí unos resultados mejores (es una condición necesaria pero no suficiente).

Las diferencias más claras en los resultados se aprecian en la esfera de la asistencia a los niños (con y 
sin discapacidad). Se constata claramente que los índices de fracaso en la vida adulta (alcoholismo y drogodependencia, conducta delictiva, suicidio) son terriblemente elevados en el caso de los niños que crecen en instituciones. Por el contrario, los adultos jóvenes que crecieron en hogares de acogida tienden a integrarse en la sociedad sin dificultades aparentes. Cada vez existen más indicios (Pinheiro, 2006: 189) que confirman que entre las consecuencias del internamiento de los niños en instituciones -aun en los casos en que las instituciones en cuestión presentan buenas condiciones materiales y un personal cualificado ${ }^{8}-$ cabe citar una salud física deficiente, graves retrasos en el desarrollo, (mayor) discapacidad y daños psicológicos potencialmente irreversibles.

\subsection{Las viviendas agrupadas frente a las viviendas dispersas}

Los entornos en forma de población (viviendas agrupadas o colectivas) pueden considerarse un modo transicional entre la asistencia institucional y la de base comunitaria. Pueden reportar ciertos beneficios a una población con un menor nivel de necesidades de apoyo y, en su caso, podrían llegar a convertirse en un elemento importante del espectro de prestación de servicios, aunque lo más previsible es que sólo lleguen a ocupar un nicho en el mercado de la asistencia.

En una reciente comparación de las viviendas agrupadas frente a las viviendas dispersas en la comunidad se puso de manifiesto que las viviendas dispersas superaban a las viviendas colectivas en la mayoría de los indicadores de calidad estudiados.

\subsection{La relación entre costes y resultados}

Los responsables políticos abordan los resultados de la asistencia institucional y de la de base comunitaria, inevitablemente, en el contexto de los costes. Las complejidades de la relación existente entre las necesidades, los costes y los resultados se abordaron con mayor detalle en el informe DECLOC. La conclusión más importante fue que, sobre la base de una comparación de las necesidades equiparables de los residentes y de una calidad de la asistencia equiparable, no existen indicios de que los modelos de asistencia de base comunitaria sean intrínsecamente más costosos que las instituciones. Otros estudios argumentan que la asistencia residencial

\footnotetext{
8 Un ejemplo típico es el de las instituciones de asistencia infantiles de algunos 'nuevos' Estados miembros que tienden a estar gestionados por los correspondientes ministerios de sanidad. Su problema no radica en las deficiencias materiales ni en la carencia de personal médico, sino en el modelo de asistencia estrictamente médica que no brinda a los menores la estimulación sensorial ni emocional necesaria para un desarrollo saludable.
}

podría ser en realidad más cara que la asistencia comunitaria (Ward, Holmes y Soper, 2008) ${ }^{9}$, pero la evaluación de todos los costes directos e indirectos -y de los beneficios a largo plazo de la creciente participación social- sigue estando abierta a interpretaciones diferentes.

Algunos responsables políticos cuyas preocupaciones son estrictamente económicas (en particular en el contexto de la crisis económica actual, véase el siguiente apartado) podrían, no obstante, sentirse más tranquilos al comprobar que la asistencia institucional de baja calidad puede resultar más barata que la asistencia comunitaria óptima que proporciona mejores resultados. No obstante, los autores del presente informe son de la opinión de que este tipo de razonamientos presentan importantes carencias. La conservación de la asistencia institucional de bajo coste y de baja calidad no constituye una opción aceptable desde un punto de vista ético y tampoco es sostenible en términos pragmáticos. Es previsible que, tarde o temprano, la presión pública provoque una mejora de la calidad de la asistencia en las instituciones donde ésta haya sido tradicionalmente deficiente y, llegados a este punto, es previsible que la asistencia comunitaria constituya una opción que, además de ser intrínsecamente mejor para el usuario, resulte más rentable desde el punto de vista del proveedor.

\subsection{El contexto de la crisis económica actual}

Los dilemas relativos a los costes y los resultados arriba indicados adquieren una nueva perspectiva a la luz de los acontecimientos más recientes. La crisis económica entraña un riesgo muy elevado de que los gobiernos (las autoridades públicas) se vean tentados a preservar su saldo presupuestario mediante la reducción del gasto destinado a servicios sociales, la mayor parte del cual corresponde a los gastos de personal. Como resultado de ello, podría aumentar el riesgo de despidos del personal de los entornos de asistencia de todos los tipos (institucionales y de base comunitaria), mientras que la calidad de la asistencia que reciben los usuarios podría verse amenazada por una reducción de costes generalizada y un descenso de la ratio entre personal y usuarios.

Desde el punto de vista del desarrollo previsible del sector de la asistencia, dichas decisiones por parte de las autoridades parecen tener poca visión de futuro. La debilitación de los estándares de asistencia que ya se han alcanzado (y que a menudo distan bastante de ser ideales), no sólo resulta éticamente

9 El estudio señalaba que el coste unitario medio del internamiento de un niño en una residencia durante una semana era 4,5 veces superior al de una modalidad de alojamiento independiente, 8 veces superior al coste de la colocación en un hogar de acogida, 9,5 veces superior al de la colocación con familiares o amigos y más de 12,5 veces superior al de la colocación con sus propios padres. 
inaceptable, sino que además contrasta enormemente con las tendencias demográficas previstas y con sus implicaciones. El sector de la asistencia constituye uno de los pocos en los que no está previsto que se registre un descenso de la demanda en el futuro; al contrario, debido al envejecimiento de la población, el número de personas que precisarán algún tipo de asistencia de larga duración tiene visos de incrementarse paulatinamente durante las próximas décadas. La reducción de costes generalizada en el sector de la asistencia y los despidos del personal cualificado se consideran por ende medidas muy contraproducentes a largo plazo debido a que entrañan el riesgo de que el personal cualificado se cambie a otros sectores, por lo que resultaría más difícil satisfacer las necesidades futuras.

Es evidente que la crisis económica no se puede ignorar. Pero para situar el proceso de la reforma adecuadamente en el contexto de la crisis económica parece necesario considerarla una oportunidad y no una excusa para demorar los procesos de transición de la asistencia institucional a la de base comunitaria existentes o previstos.

\section{Desafíos clave de la transición de la asistencia institucional a la de base comunitaria y sugerencias sobre el modo de abordarlos}

Los principales retos a los que hay que hacer frente para pasar de un sistema que depende de las instituciones residenciales a uno que brinda diversos servicios de base comunitaria repercuten sobre un amplio espectro de personas. Sin una preparación y una planificación adecuadas, los derechos e intereses de los usuarios afectados podrían quedar relegados al olvido entre las demás preocupaciones de las comunidades locales, los miembros de las instituciones y los políticos locales.

\subsection{El exceso de inversión en las modalidades insti- tucionales actuales}

Se trata de una reacción habitual en las situaciones de emergencia, principalmente en situaciones en las que se ‘detectan' situaciones en que la calidad de la asistencia es muy deficiente o en situaciones de abuso (o, peor aún, situaciones notificadas por organismos de control de los derechos humanos y por periodistas de investigación). Si bien dichas situaciones intolerables requieren una atención inmediata, existe el riesgo de que un enfoque aislado de estos casos extremos reste valor a la atención prestada a los cambios sistémicos necesarios:

- El exceso de inversión en los edificios. La mejora de las condiciones físicas de las instituciones constituye una respuesta habitual a la calidad deficiente de la asistencia en las mismas. Sin embargo, esta mejora dificulta a menudo el cierre de las instituciones a medio plazo, pues las autoridades se muestran reticentes a cerrar un servicio en el que se ha invertido una importante cantidad de dinero. Los fondos de donantes externos suelen dedicarse a menudo a este fin. Esta cuestión va más allá de la paliación de los casos extremos obvios. Incluso la asistencia de calidad media puede ser objeto de iniciativas dirigidas a mejorar los estándares materiales (a través de inversiones en el entorno físico), la ratio entre personal y usuarios u otros parámetros. Mientras que dichos cambios pueden, en algunos casos, mejorar la calidad de vida de los usuarios, este tipo de inversiones constituye a menudo la pérdida de una oportunidad de un cambio más sistémico, pues resulta más difícil abogar por el cierre y la reforma sistémica.

- Atención exclusiva a los fallos de las personas. Evidentemente, resulta necesario fomentar la responsabilidad individual de todos los miembros del personal (y, en particular, de la dirección). En los casos en que se detecten unas condiciones particularmente inaceptables y abusos de los derechos humanos, debería identificarse claramente a los responsables y, si procede, llevarlos ante la justicia. Sin embargo, no sería correcto centrarse exclusivamente en los fallos personales y dejar pasar las cuestiones sistémicas en juego. De hecho, se podría impartir formación de reciclaje a un gran número de miembros del personal que hayan formado parte de las prácticas claramente insatisfactorias a fin de brindar servicios de calidad superior en un entorno distinto.

\subsection{El riesgo de mantener servicios paralelos}

Si la acumulación de alternativas no está ligada a un cierre progresivo de las instituciones existentes, podría producirse una situación en la que los nuevos servicios de base comunitaria operen en paralelo a las instituciones preexistentes. Una parte de los usuarios permanecería en un centro de asistencia institucional sin mejoras tangibles de su condición, algo que es ya insatisfactorio de por sí y, además, podría entrañar los siguientes riesgos:

- Dejar atrás a las personas con discapacidad grave y/o necesidades complejas En los proyectos de desinstitucionalización existe una tendencia a 'hacer primero las cosas más sencillas'. Dentro de un enfoque gradual orientado al cierre de las grandes instituciones residenciales, las personas con un grado de discapacidad leve o moderado son trasladadas a servicios de base comunitaria. En muchos casos, estas personas apoyaban al personal de la institución residencial encargado de personas con un grado de discapacidad más grave. 
La desinstitucionalización de las personas de mayor nivel funcional en primer lugar origina una situación en la que el resto del personal se queda con una serie de personas con discapacidad grave para quienes no será fácil hallar plazas en el nuevo sistema de base comunitaria centrado únicamente en las necesidades de personas con menor grado de discapacidad. Estos riesgos deben evitarse mediante la plena inclusión de las personas con discapacidad grave y necesidades complejas en todas las iniciativas de desinstitucionalización desde el principio.

- Creación ('cobertura excesiva') de necesidades adicionales. Sin un traslado suficiente de usuarios de la asistencia institucional a los nuevos servicios, es muy probable (como han puesto de manifiesto los ejemplos de los Estados Unidos) que estos nuevos servicios, para justificar su existencia, intenten absorber a otros usuarios potenciales cuya necesidad de los mismos podría ser, inicialmente, reducida. Paradójicamente, incluso si se trasladan los usuarios pero se conservan las plazas en las instituciones preexistentes, dichas instituciones tienden por naturaleza a 'arrastrar' a otros usuarios potenciales, incluso pertenecientes a grupos de usuarios distintos a los previstos originalmente.

- Duplicación de gastos a largo plazo. Además, existe el riesgo de 'duplicar los gastos' de los dos sistemas que operan de forma paralela. Cabe admitir que será necesario incurrir en estos costes considerables -la inversión inicial en las nuevas instalaciones de base comunitaria para su puesta en marcha- $y$ los costes derivados de la duplicidad de operaciones para abastecer a los servicios antiguos y a los nuevos de forma paralela durante unos cuantos años hasta el cierre completo de la institución. Estos costes han de incorporarse a los presupuestos de la mano de las estrategias de desinstitucionalización. Sin embargo, ello no debería provocar una situación a largo plazo en la que ambos servicios existan de forma paralela e indefinida. Es previsible que, a largo plazo, dicho sistema de doble vertiente resultase insostenible $y$, sobre todo en épocas de crisis o de reducción de los presupuestos, las autoridades podrían optar por abolir el nuevo servicio en vez del servicio antiguo y ya consolidado. De hecho, la explotación continuada de la institución preexistente podría servir de argumento para demostrar que la desinstitucionalización en sí ha fracasado por no reducir automáticamente el número de camas en el entorno institucional.

\subsection{Alternativas excesivamente 'institucionales'}

Incluso en los casos en que se establezcan alternativas y se reduzca el tamaño de las instituciones preexistentes o recurra al cierre de las mismas, el carác- ter de estas alternativas podría resultar problemático ya que ya son excesivamente 'institucionales' de por sí, y no están basadas en las necesidades y las preferencias individuales, lo que, a su vez, se debe a menudo a una participación insuficiente de los usuarios ( $y$, en la medida de lo posible, de sus familias) en la planificación, la gestión y la evaluación de los servicios.

- Alternativas al tamaño inadecuado. Debido a la falta de entendimiento de los servicios de base comunitaria, existen casos de las llamadas políticas de desinstitucionalización, cuyas 'alternativas' son muy similares al modelo institucional. Esto podría deberse a su tamaño. Efectivamente, en algunos países incluso las instituciones relativamente grandes (con hasta 80 residentes) podrían ser el resultado final del desmantelamiento de instituciones de mayor tamaño.

- Alternativas que perpetúan la cultura institucional. Incluso en los casos en que las instalaciones alternativas son, o parecen, pequeñas, éstas podrían no alejarse lo suficiente de los centros preexistentes. En ocasiones, los nuevos servicios se organizan en las inmediaciones de la institución original, simplemente para garantizar la reutilización del personal y de los edificios. Otras veces se ofrece como alternativa el mismo edificio, tras una reorganización interna destinada a transformarlo en lo que se conoce como apartamentos 'de estilo familiar'. El resultado es que $s i$ bien se obtiene una ligera mejora de las condiciones físicas, los usuarios siguen viviendo en el mismo ambiente aislado y apenas se hace nada por su reinserción en la comunidad o por impedir nuevas admisiones.

- Alternativas con una cultura institucional dominante. Las dos opciones anteriores entrañan riesgos, en particular cuando la reforma se ve reducida a un cambio de vivienda como tal, sin una intención deliberada de impartir formación de reciclaje al personal con vistas a cambiar la cultura institucional.

\subsection{Cierre sin alternativas adecuadas}

En algunos países, las estrategias gubernamentales de desinstitucionalización fijan unas metas arbitrarias tales como 'una reducción del $50 \%$ de los usuarios de las instituciones en un período quinquenal'. Estos objetivos raramente se calculan sobre la base de la capacidad del gobierno y de las autoridades locales para desarrollar unos servicios alternativos apropiados durante dicho período de tiempo. A menudo, se consideran más bien un ejercicio de reducción de costes: las instituciones se cierran para ahorrar dinero cuando las autoridades descubren que el internamiento en centros no institucionales es más barato. 
Los donantes externos también pueden fijar unos plazos poco realistas, lo que provoca una desinstitucionalización excesivamente acelerada o un proceso incompleto, como resultado de lo cual puede perjudicarse a los usuarios que no estén preparados para trasladarse o cuyas instalaciones todavía no estén convenientemente preparadas.

Tanto si es por causa de un enfoque burocrático, de la demanda de reducción de costes o de la presión ejercida por los donantes externos, la aplicación deficiente de las políticas de desinstitucionalización puede provocar la adopción de medidas inadecuadas para cumplir el objetivo. Guardan relación con los siguientes riesgos:

- Falta de alternativas de calidad apropiada disponibles y asequibles. Cuando se fijan unas metas arbitrarias y unos plazos poco realistas (y cuando el motor subyacente es la intención de reducir costes) es habitual que no existan fondos suficientes para realizar el proceso de un modo que garantice que todos los usuarios reciban los servicios adecuados. Cabe recordar que mientras que es previsible que la asistencia de base comunitaria de la mayoría de los usuarios sea más barata que la asistencia en una institución residencial, existen ciertas categorías de usuarios que requerirán un apoyo extensivo y, por ende, más costoso (por ejemplo, atención personalizada durante 24 horas). Si estos servicios no se promueven por hacerse hincapié en la reducción de costes, estos usuarios podrían sufrir un retroceso durante el proceso de la reforma institucional. La aplicación de regímenes que apoyen a los cuidadores familiares también es indispensable para evitar dicho retroceso.

- Optar por la solución más fácil. Un resultado de los objetivos arbitrarios es que centran la atención en el traslado de los usuarios que son 'más fáciles de colocar' en la comunidad y en la reducción de los números globales en las instituciones (véase más arriba). Esta estrategia suele dejar atrás a los usuarios con mayor necesidad de apoyo, tales como los usuarios con una discapacidad grave (aunque estos usuarios con necesidades más complejas deberían haber sido prioritarios en el proceso de desinstitucionalización).

- 'Soluciones' meramente administrativas. Para lograr los objetivos arbitrarios o recortar los gastos, las autoridades locales toman a menudo la decisión de fusionar las instituciones que han reducido sus números. El resultado puede pasar por el traslado de los usuarios de un internamiento inadecuado a otro, traumatizándolos durante el proceso. Por ejemplo, los niños con discapacidad grave pueden incluso morir del shock ocasionado por un movimiento tan brusco. Las fusiones o la consolidación de las instituciones también pueden provocar una agrupación inadecuada de los usuarios (por ejemplo, la colocación de los bebés más pequeños con niños más mayores con una conducta desafiante), aumentando así el riesgo de daños y abusos. El riesgo del cuarto tipo [sic.] se ha constatado especialmente en los procesos de desinstitucionalización apresurados e improvisados en el ámbito de la asistencia de salud mental en varios países a partir de la década de los años setenta ${ }^{10}$. Aunque estos procesos reportaron, a la postre, una serie de avances positivos, con la mejora de la vida de muchos residentes antiguos, también se vieron afectados por un desarrollo paralelo de alternativas insuficiente (al menos en la fase inicial) que provocó una falta real de acceso a los servicios por parte de los usuarios.

\section{Principios básicos comunes de la transición de la asistencia institucional a la de base comunitaria}

Se ha elaborado un conjunto de principios básicos comunes basado en las mejores prácticas de la transición de la asistencia institucional a la de base comunitaria ${ }^{11}$, así como de la evaluación de los desafíos clave arriba indicados:

1. Respeto por los derechos de los usuarios y participación de los mismos en la toma de decisiones: los usuarios (y sus familias) deberían ser socios de pleno derecho durante el proceso de transición. Deberían participar activamente y ser consultados durante el desarrollo, la ejecución y la evaluación de los servicios que reciben. Los usuarios han de recibir la información adecuada de un modo en que ellos puedan comprenderla. Cuando proceda, deberían contar con el apoyo de una persona de su elección durante el proceso de toma de decisiones. Deberían respetarse las necesidades y los requisitos específicos de cada individuo. Por lo que respecta a los niños, todas las colocaciones deberían hacerse del modo que mejor sirvan a los intereses del niño.

2. Prevención del internamiento en instituciones: se han de adoptar las medidas necesarias para impedir el internamiento de individuos en instituciones $^{12}$. Es necesario contar con políticas glo-

\footnotetext{
${ }^{10}$ Estos resultados tan ambiguos fueron notificados a raíz de los procesos de desinstitucionalización de países como Italia, el Reino Unido y los Estados Unidos (Estado de Nueva York).

${ }^{11}$ Véase el informe DECLOC y el informe Second Disability High Level Group Report on Implementation of the UN Convention on the Rights of Persons with Disabilities (2009).

${ }^{12}$ En algunos ejemplos no es conveniente debido al riesgo de dañar a los usuarios, por ejemplo, en el caso de niños o ancianos víctimas de abusos o que corran el riesgo de recibir abusos en su entorno doméstico. En el caso de los niños, la mejor alternativa consiste en sustituir la asistencia familiar (colocación en hogares
} 
bales que apoyen a las familias y a otros cuidadores informales, así como el refuerzo de las capacidades de inclusión de las comunidades.

3. Creación de servicios de base comunitaria: es necesario acumular un espectro de servicios de calidad disponibles y asequibles en la comunidad para sustituir a la asistencia institucional. Dichos servicios deberían partir de las necesidades y preferencias personales de los usuarios. Deberían brindar apoyo a los cuidadores familiares e informales. Con ellos se pretende evitar que se produzcan más admisiones en los centros de asistencia institucional, facilitar la colocación de las personas que estén actualmente internadas en instituciones y también beneficiar a los que vivan en la comunidad (con sus familias o con quien corresponda), pero sin el apoyo adecuado.

4. Cierre de instituciones: este proceso debería, en la medida de lo posible, iniciarse con proyectos piloto. La planificación debería incluir la elaboración de un plan individualizado para cada usuario y la evaluación de las necesidades de formación del personal que desee trabajar en la comunidad. Debería existir una preparación apropiada de los usuarios para reducir al mínimo los riesgos de un trauma vinculado a un cambio en su entorno de vida. Las instituciones deberían cerrarse de un modo que garantice que no se deje atrás a ningún usuario en condiciones inadecuadas. Los que presenten mayores necesidades de apoyo deberían tener prioridad (por ejemplo, los bebés, los niños y los adultos con discapacidad grave o quienes estén internados en instituciones con unas condiciones físicas muy deficientes o unos regímenes abusivos).

5. Restricción a la inversión en las instituciones existentes: el proceso de transición de la asistencia institucional a la de base comunitaria suele durar varios años. Entretanto, muchos usuarios viven en condiciones inadecuadas e insalubres. Por tanto, podría ser preciso realizar alguna renovación de las instituciones existentes. Sin embargo, ésta debería limitarse a la inversión estrictamente necesaria para garantizar un saneamiento y unos servicios de agua y calefacción adecuados. Es previsible que una inversión excesiva en un edificio dificultase el cierre posterior de la instalación.

6. Desarrollo de los recursos humanos: resulta esencial garantizar la disponibilidad de personal suficiente y adecuadamente formado con las habilidades apropiadas para la asistencia de base comunitaria, la cual se fundamenta en asociaciones, en actitudes integradoras y en un enfoque interdisciplinar. Ello podría conllevar un reciclaje y una recualificación del personal que antes trabajaba en el contexto de la cultura institucional. Es posible reducir la resistencia al proceso de reforma mediante la redistribución del personal que puede ser formado para trabajar en la comunidad. Es aconsejable contar con la participación de representantes del grupo de usuarios en calidad de formadores durante la cualificación y la recualificación del personal de todos los niveles. También debería permitirse la participación de los cuidadores familiares en los programas de formación destinados a mejorar la calidad de la asistencia prestada.

7. Uso eficiente de los recursos: el sistema institucional actual conlleva una enorme asignación de recursos: presupuestos, edificios y equipos. En la medida de lo posible, $y$ en pro de los intereses de los usuarios, estos recursos deberían transferirse de la institución existente a los nuevos servicios. Mediante la reutilización de los recursos existentes se garantiza que el proceso de reforma resulte más barato y más sostenible. Los presupuestos correspondientes a los costes de explotación pueden ser transferidos para cubrir los costes de la explotación de los servicios en la comunidad, tales como los hogares para pequeños grupos y los centros de apoyo familiar; en ocasiones, los edificios pueden reutilizarse para otros fines (cuando estén adecuadamente ubicados y en unas condiciones suficientemente buenas).

8. Control de la calidad: los sistemas de control de la calidad deberían guardar relación con el proceso de transición y con los servicios resultantes, con un enfoque claramente definido y orientado a la satisfacción de los usuarios. La participación de estos últimos, de sus familias y de las organizaciones que los representen, en la vigilancia de la calidad, es crucial.

9. Enfoque global: en todas las esferas políticas relevantes, entre ellas el empleo, la educación, la salud, la política social, etc., surgirán cuestiones concernientes a la transición de la asistencia institucional a la de base comunitaria que habrá que abordar. El enfoque global servirá para garantizar la coordinación y la coherencia política en todas las ramas gubernamentales y en la continuidad de la asistencia, por ejemplo, entre la niñez y la edad adulta ${ }^{13}$.

Sensibilización permanente: el proceso de transformación debe ir de la mano de una serie de iniciativas que garanticen el apoyo de los órganos profesionales clave en lo referente a los valores que transmiten a sus miembros actuales y potenciales,

\footnotetext{
${ }^{13}$ Esto es especialmente aplicable a los individuos con necesi-
} rarse las opciones de carácter residencial cuando estas alternativas no sean viables.

de acogida y adopción, según convenga) y sólo deberían conside- 
así como a la sociedad en su conjunto. Simultáneamente, debería fomentarse la sensibilización de los encargados de la toma de decisiones y los creadores de opinión y del público en general a fin de garantizar la coherencia de sus actitudes con los valores deseados. Es preciso prestar una atención especial al desarrollo de actitudes integradoras en las comunidades locales, en aquellos lugares donde se vayan a poner en marcha los servicios.

\section{Recomendaciones}

Al abordar las cuestiones derivadas de la reforma de la asistencia institucional, tanto los Estados miembros como las instituciones de la UE deberían respetar y hacer valer los derechos y los principios recogidos en la Carta de los Derechos Fundamentales de la Unión Europea. Además, debe prestarse la debida atención a las obligaciones derivadas de los correspondientes tratados internacionales sobre derechos humanos: el Convenio Europeo para la Protección de los Derechos Humanos y de las Libertades Fundamentales (CEDH), el Convenio Europeo para la prevención de la tortura y de las penas o tratos inhumanos o degradantes, el Pacto Internacional de Derechos Económicos, Sociales y Culturales (PIDESC), la Convención sobre los Derechos del Niño (CDN) y el Convenio internacional sobre la eliminación de todas las formas de discriminación racial ${ }^{14}$, así como la nueva Convención sobre los Derechos de las Personas con Discapacidad que será ratificada sin demora por los Estados miembros y por la Comunidad Europea.

\subsection{Recomendaciones dirigidas a los Estados miembros}

- Revisión y modificación de las normas jurídicas y administrativas a fin de garantizar la participación activa de los usuarios de los servicios, incluidos los niños, en los procesos de toma de decisiones que les afecten y también en el diseño de los servicios.

- Identificación de las normas jurídicas y administrativas que apoyan directa o indirectamente el internamiento en instituciones o bloquean la transición a la asistencia de base comunitaria y modificación de las mismas a fin de apoyar la prestación de unos servicios de calidad en la comunidad.

- Adopción de estrategias y planes de acción basados en los Principios Básicos Comunes, acompañadas de un calendario de plazos y un presupuesto claramente definidos para el desarrollo

\footnotetext{
${ }^{14}$ Este convenio es especialmente relevante en el contexto de una sobrerrepresentación del pueblo romaní en las instituciones dedicadas a la asistencia infantil en varios Estados miembros.
}

de los servicios en la comunidad y el cierre de las instituciones residenciales de estancia prolongada. Elaboración de un conjunto de indicadores apropiados destinado a medir el grado de aplicación de estos planes de acción.

- Utilización de los Fondos Estructurales para la transición de la asistencia institucional a la de base comunitaria. El Fondo Social Europeo puede aportar financiación para la formación (y el reciclaje) del personal y el Fondo Europeo de Desarrollo Regional (FEDER) puede utilizarse simultáneamente para el desarrollo de una infraestructura social de apoyo a los nuevos servicios de base comunitaria ${ }^{15}$. Los Estados miembros deberían asegurarse de que los fondos del FEDER no se utilizan para construir nuevas instituciones residenciales segregadoras y de que su utilización para la mejora de la infraestructura de las instituciones existentes, cuando ésta esté permitida, esté vinculada a la inversión en una reforma sistémica de la asistencia y no sobrepase el 10\% de los gastos totales.

- Establecimiento de sistemas de vigilancia obligatoria de la calidad relacionados con la calidad de vida de los usuarios en vez de unos indicadores de los insumos. Estos sistemas deberían estar basados en la participación de los usuarios, sus familias y las organizaciones que los representen, en el proceso de vigilancia.

- Fomento de la mejora de las condiciones de trabajo de los cuidadores profesionales, con el objeto de hacer que los puestos de trabajo de este sector resulten más atractivos. Obligación de que los organismos que representen, formen $y$ acrediten el ejercicio profesional del personal que trabaje con ancianos, niños, personas con problemas de salud mental y personas con discapacidad adquieran un compromiso de apoyo a la dignidad humana, la inclusión y la autonomía de los usuarios de los servicios en su trabajo. Aseguramiento de que la formación y la acreditación del personal incluyan a los usuarios de los servicios y estén basadas en el principio de inclusión.

- Dotación de un apoyo sistemático a los cuidadores informales (especialmente a los cuidadores familiares) a fin de garantizar la calidad de la asistencia que presten así como de garantizar su propia calidad de vida.

- Garantía de la coordinación de los diferentes departamentos y agencias gubernamentales que participen en el proceso de transición. El éxito de la aplicación de los planes de reforma pasa por

${ }^{15}$ A comienzos de 2009, la Comisión Europea presentó un ejercicio conjunto con Bulgaria orientado a la utilización de los Fondos Estructurales (tanto los del FEDER como los del FSE) para la construcción de centros de asistencia comunitarios, facilitando el proceso de institucionalización. Este ejemplo podría repetirse en otros Estados miembros. 
una coordinación firme entre todos los actores relevantes, tanto a nivel horizontal (varios ministerios, tales como los ministerios de sanidad y asuntos sociales) como vertical (autoridades nacionales y locales).

\subsection{Recomendaciones dirigidas a la Comisión Europea}

- Presentación de una Comunicación relativa a la transición de la asistencia institucional a la de base comunitaria que incluya los Principios Básicos Comunes que se vayan a aplicar durante el proceso de transición.

- Incorporación de los Principios Básicos Comunes en todas las políticas comunitarias relevantes (por ejemplo, la política sobre discapacidad, protección social e inclusión social, empleo, igualdad de oportunidades, desarrollo regional, sanidad, educación, investigación, derechos del niño y relaciones exteriores). En particular, incorporación de dichos principios en los siguientes instrumentos políticos:

- La próxima Estrategia Europea en materia de Discapacidad (a partir de 2010) que proporcionará un marco de coordinación a fin de contribuir a la aplicación de la Convención sobre los Derechos de las Personas con Discapacidad de las Naciones Unidas en el ámbito de la UE y de los Estados miembros, incluido el derecho de todas las personas con discapacidad a vivir de forma independiente y a ser incluidas en la comunidad, y apoya los compromisos voluntarios adquiridos por los Estados miembros y la creciente cooperación entre estos últimos y la Comisión.

- El método abierto de coordinación en el ámbito de la protección social y la inclusión social, que fomenta el aprendizaje conjunto y la mejora de las iniciativas de los Estados miembros orientadas a la transición de los servicios institucionales a los de base comunitaria ${ }^{16}$. Es preciso pedir a los Estados miembros que faciliten información más específica, incluidos las cifras de personas internadas en instituciones y de las que reciben asistencia en el hogar o de base comunitaria. Es preciso identificar a las personas que abandonen la asistencia institucional entre los grupos objetivo prioritarios de la estrategia de inclusión activa.

- La Estrategia sanitaria de la UE, en las iniciativas relevantes tales como las relacionadas con el envejecimiento saludable y la enfermedad de Alzheimer y otras enfermedades mentales,

${ }^{16}$ Como se subraya en el Informe conjunto sobre la protección e inclusión social 2009, de la UE. con las conclusiones extraídas de la consulta correspondiente al Libro Verde sobre el personal sanitario europeo y los acontecimientos destinados a aplicar el Pacto Europeo por la Salud Mental y el Bienestar.

- Elaboración des directrices relativas a la utilización de los Fondos Estructurales y otros instrumentos de financiación de la UE para la desinstitucionalización. En estas directrices cabría incluir ejemplos de buenas prácticas relacionados con el modo en que los Fondos Estructurales y otros instrumentos de financiación de la UE pueden ayudar a los proyectos sobre el desarrollo de alternativas de base comunitaria a las instituciones, por medio de la identificación de unos procedimientos y de unos actores principales. Es necesario aclarar que los proyectos destinados a construir, ampliar o perpetuar las instituciones no son coherentes con la Convención sobre los Derechos de las Personas con Discapacidad ni con las políticas de la UE relativas a la igualdad de oportunidades, inclusión social y discriminación, y no cumplen por tanto los requisitos de financiación.

- Fomento y facilitación del intercambio de modelos de buenas prácticas, por ejemplo, mediante la asignación de presupuestos destinados al aprendizaje permanente para que los gestores y los promotores de los servicios puedan ampliar sus conocimientos en lo referente a los casos de éxito. Facilitación de la elaboración de módulos de formación destinados al personal de primera línea y a los gestores sobre la conversión de la asistencia institucional al apoyo comunitario. El Programa de Aprendizaje Permanente (Leonardo o Grundtvig) podría proporcionar presupuestos disponibles.

- Creación de un panel de expertos independientes en materia de desinstitucionalización que pueda prestar asistencia técnica a la propia Comisión y, sobre todo, a los Estados miembros a la hora de asignar los recursos de los Fondos Estructurales. Para la creación de este panel de expertos (incluidas todas las partes interesadas) se recomienda utilizar los fondos del presupuesto destinado a la asistencia técnica dentro del marco de los programas operativos.

- Mejora de la base de conocimientos mediante la definición -en colaboración con EUROSTAT y con los Estados miembros- de un conjunto mínimo de datos relacionados con los servicios residenciales que permita la vigilancia del progreso de los procesos de cierre de las instituciones y el desarrollo de servicios en la comunidad, así como la comparación de los datos a lo largo de toda la UE. Deberían publicarse las estadísticas relativas al progreso alcanzado en cada Estado miembro en la transición de las instituciones a las alternativas de base comunitaria. 
- Solicitud de recopilación de datos, investigación y análisis a la Agencia de los Derechos Fundamentales dentro del marco de su mandato.

- Financiación de la investigación que facilitará la transición de las instituciones a los servicios de base comunitaria, la cual puede incluir un estudio sobre el modo de apoyar a los usuarios en el proceso de elección de sus modos de vida y la celebración de contratos de apoyo jurídicamente vinculantes con los proveedores de servicios en aplicación del artículo 12 del Convenio, así como un análisis jurídico de la legislación de los Estados miembros que fomente, directa e indirectamente, el internamiento en instituciones.

- Sensibilización dentro de la Comisión Europea (acerca del derecho a vivir de forma independiente en la comunidad y la necesidad de desarrollar un servicio de base comunitaria) entre las distintas Direcciones Generales y entre las unidades geográficas relevantes que se encarguen de los Fondos Estructurales a través de distintos medios como por ejemplo la formación, los talleres o las publicaciones.
- Inclusión de la cuestión de la inversión en la asistencia de base comunitaria en el debate acerca del futuro de la Política de Cohesión a partir de 2013.

- Tratamiento de la cuestión de la asistencia institucional y su reforma dentro del contexto de las políticas de ampliación, vecindad y desarrollo. Deberían utilizarse informes sobre el progreso para señalar las medidas que deberán adoptar los países candidatos, los países candidatos potenciales y terceros países para fomentar el desarrollo de alternativas de base comunitaria a las instituciones y la inclusión social de los ancianos, los niños, las personas con problemas de salud mental y las personas con discapacidad. Para que la vigilancia de la aplicación de sus recomendaciones resulte más eficaz, la Comisión debería colaborar con las organizaciones que representen a los usuarios en dichos países. La Comisión debería asegurarse de que la financiación europea en estos países se destine a promover el desarrollo de alternativas de base comunitaria a las instituciones. 